\title{
ATV GNC FLIGHT PERFORMANCE AND LESSONS LEARNED
}

\author{
O. Mongrard ${ }^{1}$, B. Cavrois ${ }^{2}$, F. Ankersen ${ }^{1}$, \\ O. Dubois-Matra ${ }^{1}$, M. Zink ${ }^{2}$, A. Vergnol ${ }^{2}$, \\ E. Piquemal ${ }^{2}$, G. Pionnier ${ }^{2}$, and U. Southivong ${ }^{2}$ \\ ${ }^{1}$ ESA-ESTEC \\ Noordwijk, Netherlands \\ ${ }^{2}$ Airbus Defence and Space \\ Les Mureaux, France
}

ESA's fifth and final Automated Transfer Vehicle (ATV), Georges Lemaître, performed its fully automated rendezvous and docking with the International Space Station (ISS) on August 12, 2014. The ATV's navigation sensors have shown their worth docking the 20-ton vehicles with aft port of the Space Station, manoeuvring into position and docking with an excellent accuracy. For the second consecutive time after ATV-4, the accuracy at docking was such that the ATV probe head was directly captured inside the Zvezda docking mechanism without contact with the receiving cone. From $30 \mathrm{~km}$ and down to a distance of $250 \mathrm{~m}$, ATV uses GPS (Global Positioning System) information from its own receiver and the Station's that is transmitted over a radiofrequency link. As it moves closer, ATV switches to laser navigation, using the reflection of laser pulses on reflectors mounted on the Space Station. This paper presents the achievements and performance of ATV GNC (Guidance, Navigation, and Control) across the 5 missions for both types of navigation. It will also discuss the observations made during the various flights regarding unforeseen conditions such as space environment or target pattern contamination having a potential impact on performance and how they were resolved.

\section{INTRODUCTION}

The last ATV performed a controlled reentry over the Pacific Ocean on February 15, 2015, marking the end of the ESA ATV Programme. The ATV Programme has got no equals in the ESA history for technical and programmatic

(C) The Authors, published by EDP Sciences. This is an open access article distributed under the terms of the Creative Commons Attribution License 4.0 (http://creativecommons.org/licenses/by/4.0/). 


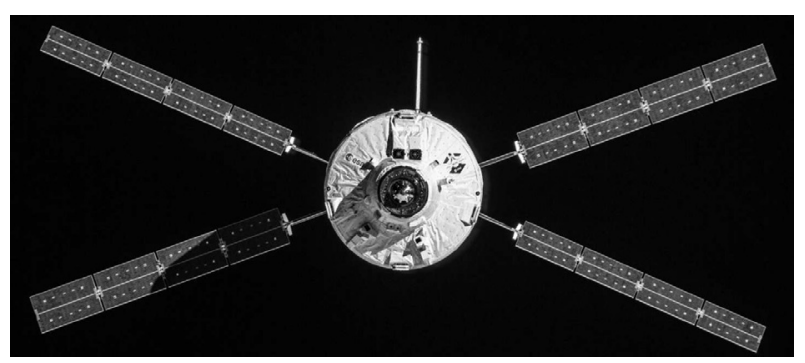

Figure 1 ATV-4 prior docking to ISS

complexity as well as level and duration of the engaged resources. With 5 successful ATV cargo missions to the ISS, over seven years, the European spaceship has demonstrated its reliability as the largest and most versatile ISS cargo vehicle. It has been acknowledged by the ISS partners as a robust and extremely capable vehicle. Automated transfer vehicle had the largest upload capability of all ISS visiting vehicles, as it can carry nearly $7 \mathrm{t}$ of dry and fluid cargo including water, gas, ISS refueling propellant but also food and new experiments or spares. Together with the Russian Progress, the ATV during its 6-month stay at the ISS was the only visiting vehicle that can perform ISS refueling, ISS attitude control, ISS reboost, and - whenever needed — debris avoidance manoeuvres. The ATV was a multifunctional spaceship, combining the automatic capabilities of an unmanned vehicle with human spacecraft safety requirements (Fig. 1). New rendezvous technologies had been developed in the frame of the ATV programme to ensure such automated and safe docking to a manned facility. These new developments paved the way for the other ISS visiting vehicles.

This paper provides an overview of the performance of the ATV flight control during the automated rendezvous phase, comparing the 5 missions. Across the 5 ATV missions, some anomalies have been encountered and resolved. The last part of the paper intends to provide some lessons learnt or recommendations based on this experience.

\section{ATV RENDEZVOUS GNC PERFORMANCE}

Automated transfer vehicle rendezvous is initiated about $30 \mathrm{~km}$ behind ISS along the local velocity vector and $5 \mathrm{~km}$ below along nadir as illustrated in Fig. 2. At such distance, the Proximity Link with ISS can be established and relative navigation using ATV and ISS GPS receiver data (Fig. 3) initialized. Relative GPS (RGPS) navigation is used by ATV up to a station keeping point located $250 \mathrm{~m}$ behind ISS Zvezda service module. To meet its safety requirement toward ISS, 


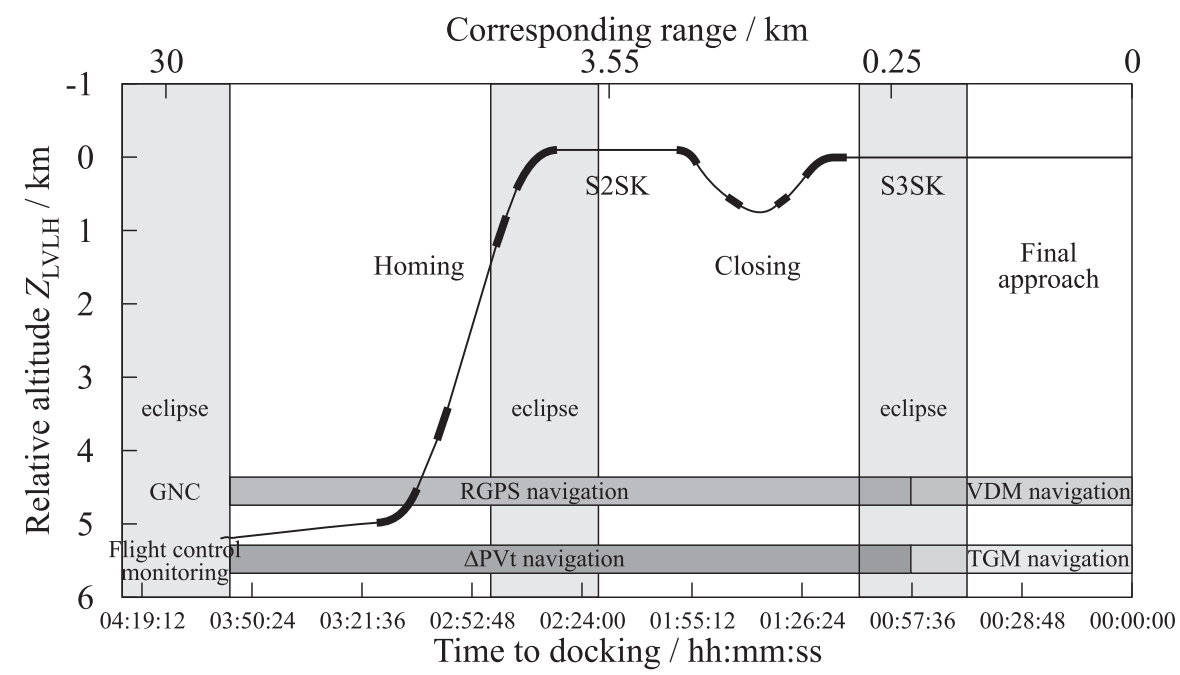

Figure 2 The ATV-5 rendezvous relative trajectory along $Z_{\mathrm{LVLH}}$ as a function of time to docking (bold black arcs represent the boost phases)

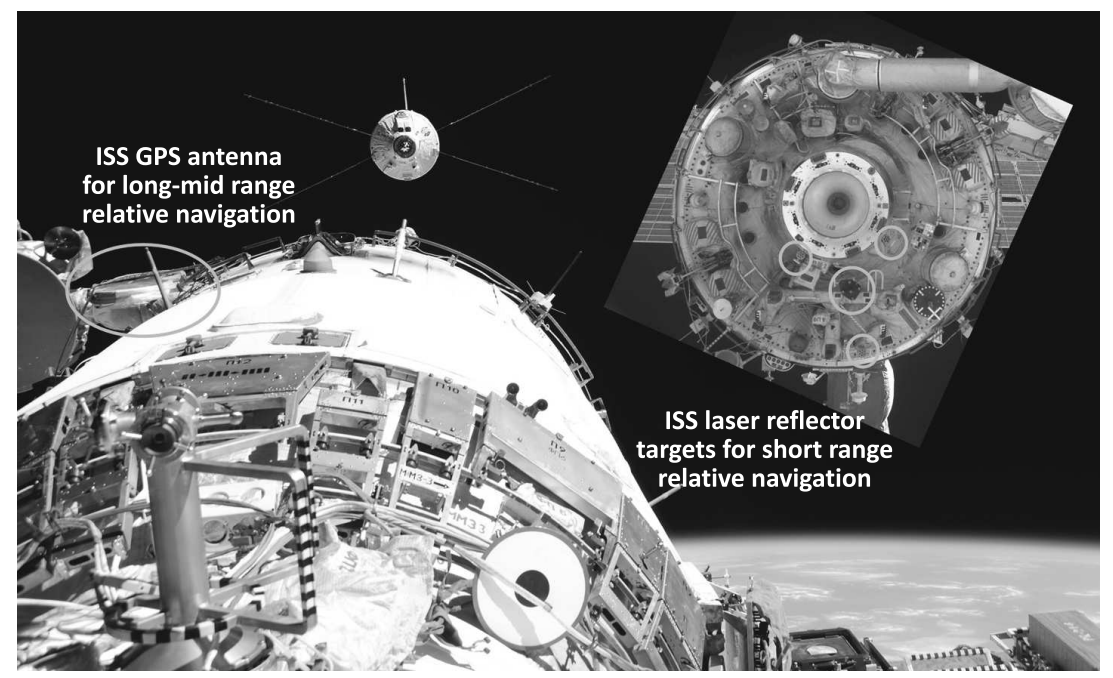

Figure 3 The ISS navigation hardware used for ATV rendezvous; GPS receiver for navigation up to S3SK and laser reflector target pattern for final approach 
ATV implements both the nominal GNC [1] function that is steering the vehicle toward docking with ISS and on top a dedicated Flight Control Monitoring function [2] that performs an independent monitoring of the trajectories to ensure safety [3]. During the far rendezvous, the GNC used relative GPS navigation [4] that is processing raw GPS data from common GPS spacecraft observed by ISS and ATV receivers while Flight Control Monitoring (FCM) navigation relies on the comparison of the Position, Velocity, and time (PVt) solution of each receiver. Having these two independent navigations being performed onboard allows to make comparison to assess their respective performance as will be described in the following sections.

During the close rendezvous or final approach, ATV performs a translation along $X_{\mathrm{LVLH}}$ from S3 station keeping up to $20 \mathrm{~m}$ and then following a fly-around maneuver aligns itself with the ISS docking port axis for the final translation toward the docking port at a range rate of $6 \mathrm{~cm} / \mathrm{s}$. During this phase, the nominal sensor used by GNC is the ViDeoMeter (VDM), while FCM uses the TeleGonioMeter (TGM) for monitoring. Both types of sensors are used in hot redundancy with a redundant equipment for each rendezvous. All sensors are using a unique optical target pattern composed of multiple retroreflectors and installed on the aft port of ISS service module (see Fig. 3) to perform navigation measurements. More details over the rendezvous scenario and ATV Flight Control can be found in $[5,6]$.

\subsection{Far Rendezvous}

For all flights, the initial convergence of the RGPS filter during the initialization of the rendezvous has been much better than the specified $10 \mathrm{~min}$. The convergence was reached within about $1 \mathrm{~min}$ for ATV-1 and ATV-2, 2 min for ATV-3 and ATV-4, and 3 min $30^{\prime}$ for ATV-5. The variability of the convergence time is expected based on the specific GPS constellation configuration at the beginning of the rendezvous and the accuracy of the initialization performed by ATV Control Center.

There is no possible comparison with a "perfect" reference to finely estimate the performance of the GPS navigation filter, but the comparison between the RGPS navigation and the $\triangle \mathrm{PVt}$ navigation provides valuable information on the estimation capability.

Two graphs in Fig. 4 present the estimation of position performed onboard by RGPS and $\triangle \mathrm{PVt}$ for ATV-2 to ATV-5. Data start with Pre-Homing and finish with S3 Station Keeping (S3SK) and has been synchronized among flights with respect to equator crossing. Note that ATV-1 is not shown on these figures due to the fact that it is not directly comparable since the ISS GPS receiver was implementing some ionospheric corrections which were suppressed in subsequent flights. The plots show a similar and consistent behavior across the 


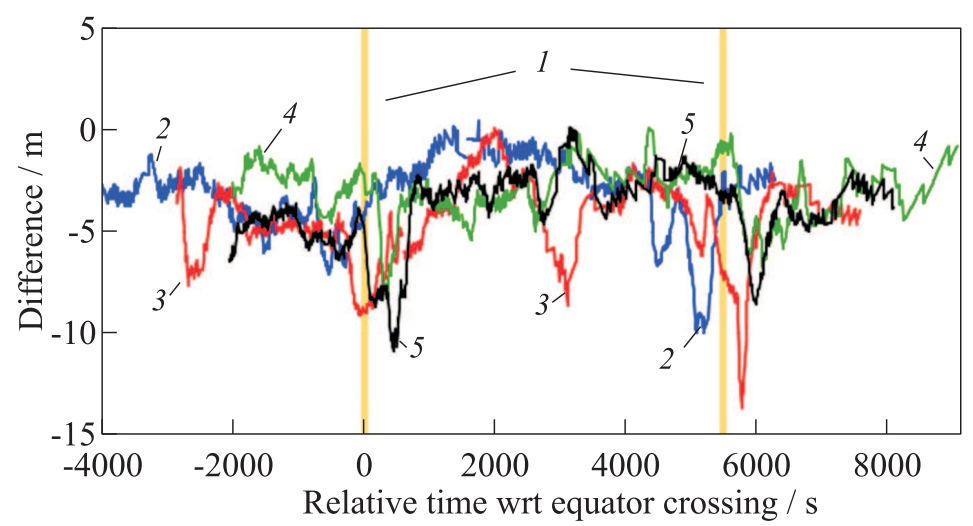

(a)

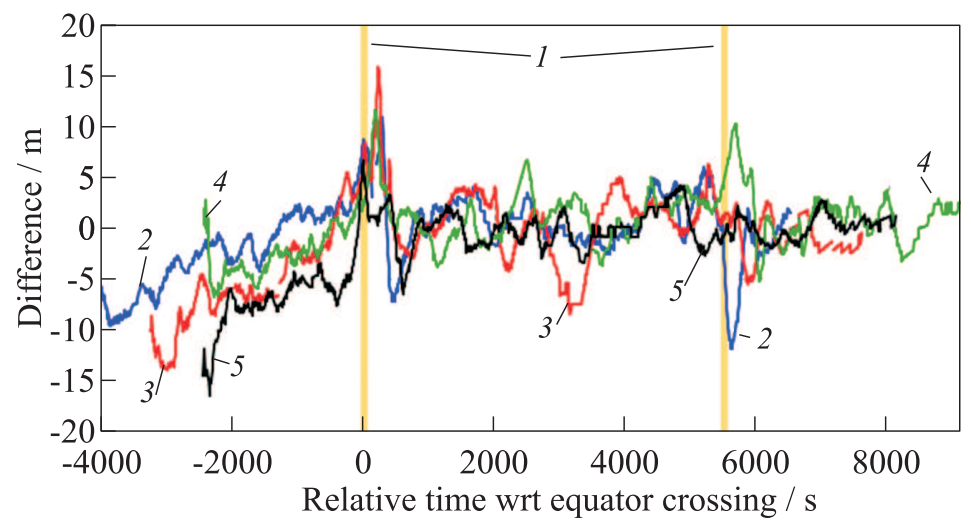

(b)

Figure 4 Position difference (RGPS- $\Delta \mathrm{PVt}$ ) along $X_{\mathrm{LVLH}}(a)$ and $Z_{\mathrm{LVLH}}(b): 1-$ equator; 2 - ATV-2; 3 - ATV-3; 4 - ATV-4; and $5-\mathrm{ATV}-5$

various flights. During the initial phase, there is a slight decreasing bias on the radial component $Z_{\mathrm{LVLH}}$ that can be explained by the difference in definition of the relative frames used by RGPS and $\triangle \mathrm{PVt}$ filters that is observable at large distance $(\sim 30 \mathrm{~km})$. Otherwise, both navigations are consistent with each other within less than $5 \mathrm{~m}$ for both $X_{\mathrm{LVLH}}$ and $Z_{\mathrm{LVLH}}$ components during almost all the rendezvous, but for some specific peaks of 10 - to 15 -meter magnitude. In velocity, the consistency between the two navigations is within $0.02 \mathrm{~m} / \mathrm{s}$ of each others outside of the peaks.

The $\Delta \mathrm{PVt}$ algorithm does not perform any consistency control on the selection of the satellites taken into account to compute PVt solutions. For in- 


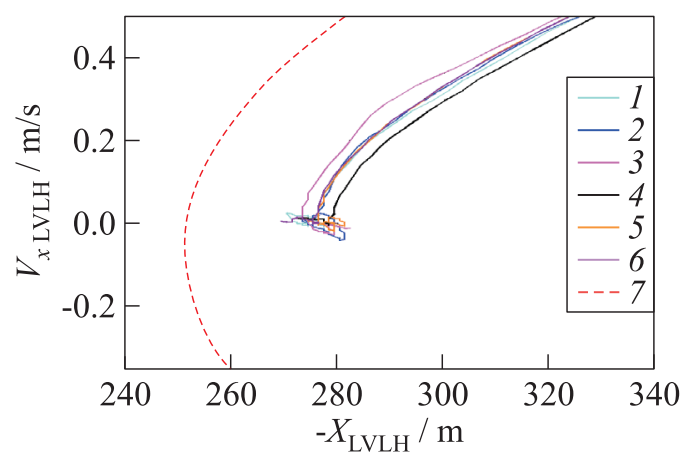

Figure $5 \Delta$ PVt $V_{x}$ monitoring - closing and S3SK trajectories in $V_{x}-X$ plane: $1-$ JV DD2 (Jules Verne during demoday 2); 2 - JV docking; 3 - JK docking; 4 - EA docking; 5 - AE docking; 6 - GL docking; and 7 - threshold

stance, it was confirmed that satellites can be used by ISS GPS receiver and not by ATV GPS receiver. This phenomenon leads to some discrepancy between ATV PVt and ISS PVt, which is greatly amplified by the ionosphere effect. Figure 4 shows the correlation between the equator crossing during sunlight by the ATV and the observed peaks in difference between RGPS and $\triangle \mathrm{PVt}$. This confirms the influence of the ionosphere effect on the observed peaks.

In conclusion, the observed peaks are due to $\Delta \mathrm{PVt}$ increase error due to the combination of the ATV GPS and ISS GPS tracking list difference and the ionosphere effect at the equator.

For all flights, the trajectories are within less than $10 \mathrm{~m}$ of each other in the final phase of the far rendezvous as can be seen in Fig. 5. A margin of about $20 \mathrm{~m}$ was always preserved with respect to onboard safety barriers [3] (dashed line threshold in Fig. 5). In general, the far rendezvous trajectories of all flights are comparable and well within the defined trajectory safety thresholds.

The robustness of ATV GNC was particularly illustrated during ATV-4 far rendezvous when a pressure sensor anomaly in a thruster triggered the isolation of one propulsive chain during the first boost of the Homing sequence (see Fig. 2). This event led to the modification, within the next commanding cycle, of the propulsive configuration and, hence, thrusters available for the Thrusters Management Function (TMF) [7] to implement GNC commands. Automated transfer vehicle was designed to ensure mission continuation after a first failure and that incident had no impact on either the boost accuracy or the attitude controller. ATV-4 pursued the remaining part of the rendezvous on the alternative propulsion configuration without any performance degradation. 


\subsection{Close Rendezvous}

The range-rate vs. range profile from the S3SK point up to docking has been followed in a very similar and consistent way by all ATVs as can be seen in Fig. 6 . After an initial acceleration phase leaving S3 up to a range rate of about $0.5 \mathrm{~m} / \mathrm{s}$, ATV gradually decelerates to reach the final translation range rate of $\sim 0.6 \mathrm{~m} / \mathrm{s}$. A slight controller overshoot is visible at the transition from the acceleration phase to the linear deceleration phase. It is similar for all spacecraft. The station keeping points in $S_{4}$ at $20 \mathrm{~m}$ and $S_{41}$ at $12 \mathrm{~m}$ are also visible on that chart as range rate is then temporary brought down to zero.

The range difference between the VDM sensor used by GNC and the TGM used by FCM provides an insight on the respective performance on the navigation during the final approach. Figure 7 shows the difference from $S_{3}$ up to docking for all 5 ATV missions. Above $200 \mathrm{~m}$, there is a clear bias between the two sensors of up to $2-3 \mathrm{~m}$ that is due to VDM range measurement performance. Closer than $200 \mathrm{~m}$, the difference between the two measurements remains smaller than $1 \mathrm{~m}$ and is decreasing with range. Below $50 \mathrm{~m}$, the range provided by the two sensors is within $0.1 \mathrm{~m}$ of each other, except for a small peak visible on all flights at $30 \mathrm{~m}$ due to a specific diffraction effect seen only by VDM-1 at such distance and still within specified range performance.

During the ATV final approach, ISS is using its thrusters to maintain its attitude around an equilibrium point close to LVLH (local vertical, local horizontal). Table 1 shows the average attitude profile of ISS during all ATV mission. Except for ATV-1, for which ISS assembly was not completed, the attitude profile followed by ISS has been similar. In Fig. 8, the temporal evolution of ISS attitude in yaw, pitch, and roll during ATV-5 final approach is reproduced. It can be

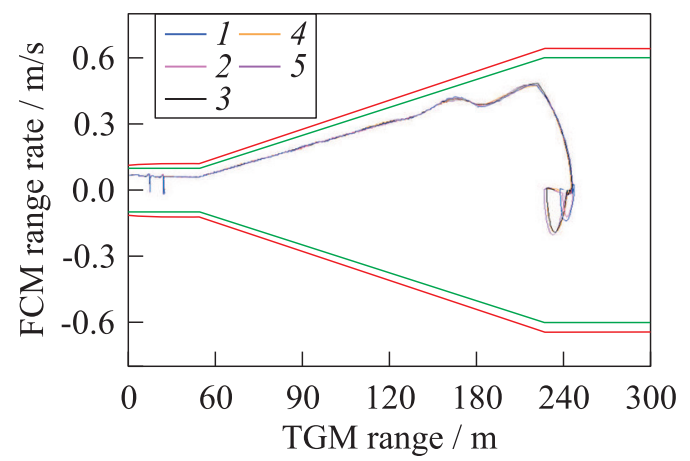

Figure 6 Range rate monitoring - final approach trajectories in range-rate vs. range: 1 - ATV1-JV; 2 - ATV2-JK; 3 - ATV3-EA; 4 - ATV4-AE; and 5 ATV5-GL 


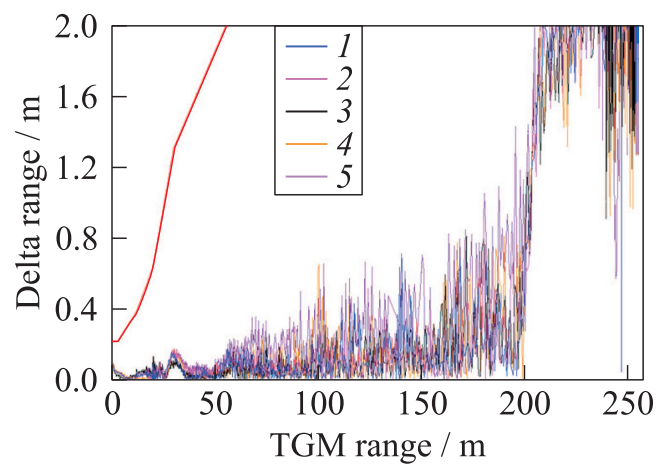

Figure 7 Delta range between TGM-1 and VDM-1 during final approach: 1 ATV1-JV; 2 - ATV2-JK; 3 - ATV3-EA; 4 - ATV4-AE; and 5-ATV5-GL

Table 1 Synthesis table - ATV final approach

\begin{tabular}{|c|c|c|c|c|c|}
\hline & \multirow{2}{*}{$\begin{array}{c}\text { ISS } \\
\text { average } \\
\text { attitude } \\
\text { [yaw; pitch; roll] }\end{array}$} & \multicolumn{2}{|c|}{$\begin{array}{c}\text { Fly-around PH motion, } \\
\mathrm{cm}\end{array}$} & \multirow{2}{*}{$\begin{array}{c}\text { Transverse } \\
\text { position } \\
\text { at contact, } \\
\mathrm{mm}\end{array}$} & \multirow{2}{*}{$\begin{array}{c}\text { Time between } \\
\text { first contact } \\
\text { and capture, } \\
\text { ms }\end{array}$} \\
\hline & & $\begin{array}{c}Y \\
\text { axis }\end{array}$ & $\begin{array}{c}Z \\
\text { axis }\end{array}$ & & \\
\hline ATV1-JV & {$\left[-0.6^{\circ} ; 0.6^{\circ} ; 0.6^{\circ}\right]$} & 50 & 40 & 7.7 & $760(\sim 17 \mathrm{~mm})$ \\
\hline ATV2-JK & {$\left[0.6^{\circ} ; 0.6^{\circ} ;-0.6^{\circ}\right.$} & 0 & 37 & 3.7 & $500(7-10 \mathrm{~mm})$ \\
\hline ATV3-EA & {$\left[0.6^{\circ} ; 0.6^{\circ} ;-0.6^{\circ}\right.$} & 10 & 40 & 2.6 & $400(<10 \mathrm{~mm})$ \\
\hline ATV4-AE & {$\left[0.6^{\circ} ; 0.6^{\circ} ;-0.6^{\circ}\right]$} & 15 & 37 & 2.2 & Direct capture \\
\hline ATV5-GL & {$\left[0.6^{\circ} ; 0.6^{\circ} ;-0.6^{\circ}\right.$} & 12 & 30 & 2.0 & Direct capture \\
\hline
\end{tabular}

seen that ISS maintains its attitude within $0.1^{\circ}$ of the reference. Hence, the ISS attitude motion encountered in flight is very benign compared to the worst case attitude evolution considered for ATV qualification. The two-way motion specified for ISS worst case attitude motion during rendezvous is reproduced by 1 in Fig. 8 and can be compared to flight data. Therefore, the ATV GNC controller design [8] that considered such motions for its qualification is very robust and handles the smooth ISS attitude evolution with ease.

From the station keeping point in $\mathrm{S} 4$ at about $20 \mathrm{~m}$ from docking port, ATV starts aligning itself with the ISS docking port. The position of the probe head at the extremity of ATV docking port is then maintained along the ISS docking port axis. Figure 9 shows that after the transition from LVLH to ISS docking axis, the probe head has been kept aligned with a good accuracy for all flights during the last $20 \mathrm{~m}$. Despite the ISS attitude motion, the transverse motion (TM) of 




Figure 8 The ISS attitude with respect to LVLH during ATV-5 rendezvous in yaw/pitch/roll (1 - two-way motion) compared to specification used for ATV qualification: 2 - yaw, TM; 3 - pitch, TM; and 4 - roll, TM

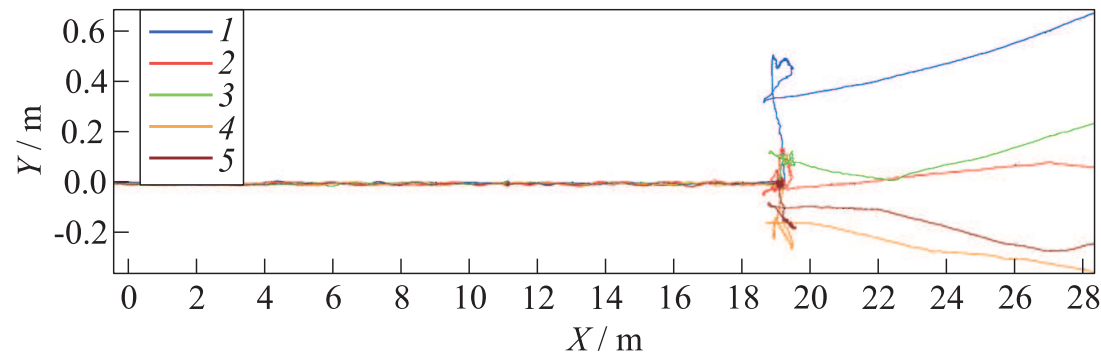

(a)

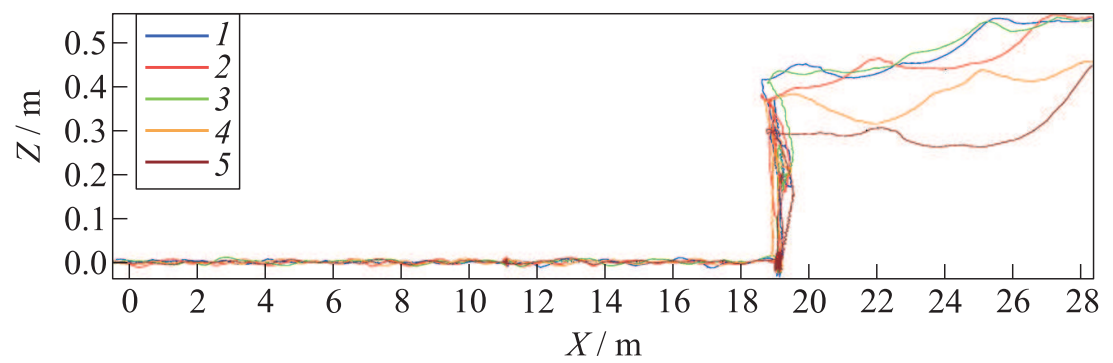

(b)

Figure 9 Estimated ATV probe head position in ISS frame during last $30 \mathrm{~m}: 1-$ ATV-1;2-ATV-2;3-ATV-3;4-ATV-4; and 5-ATV-5 


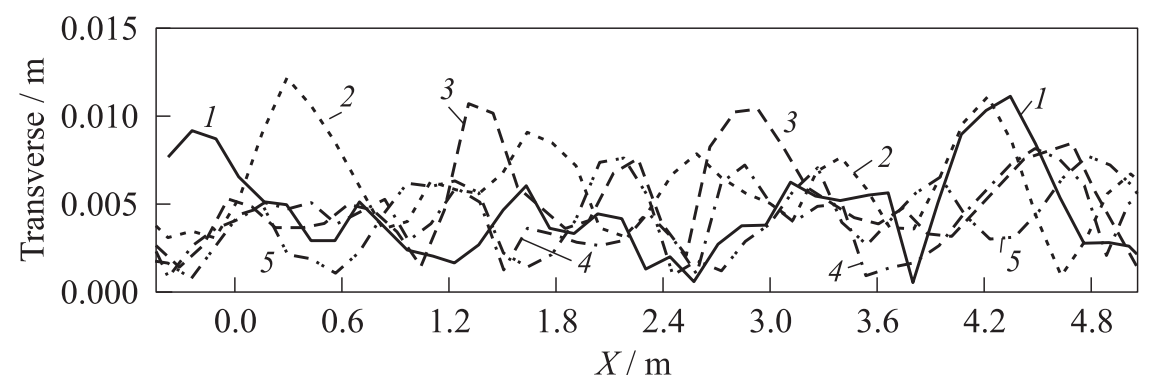

Figure 10 Estimated ATV probe head position in ISS frame, zoom on last $5 \mathrm{~m}: 1-$ ATV-1; 2 - ATV-2; 3 - ATV-3; 4 - ATV-4; and 5 - ATV-5

the probe head with respect to the ISS docking axis remains within about $1 \mathrm{~cm}$, which is much better than the 10-centimeter error specified at docking.

A zoom on the last $5 \mathrm{~m}$ shows that the transverse position of the probe head is indeed within $1 \mathrm{~cm}$, and even within $0.5 \mathrm{~cm}$ when entering the docking cone (range equals zero) for all flights but ATV-1. The accuracy at docking can be measured by the transverse probe head position at first contact and crosschecked vs. time between the first contact of the ATV probe head and the ISS docking cone and the capture of the probe head inside the receiving cylinder in the centre of the docking cone. While for the first three ATVs the probe head touched first the receiving cone before being pushed inside the cone for capture within 0.76 to $0.4 \mathrm{~s}, \mathrm{ATV}-4$ and ATV-5 achieved direct capture of the probe inside the docking mechanism (see Table 1). It means the probe head touched directly the center of the docking mechanism within only a couple millimeters. The VDM measurements estimated the transverse position to about $2 \mathrm{~mm}$ for both flights (Fig. 10).

This extreme accuracy for such a large vehicle reached twice in a row illustrates the high performance of the ATV GNC but also the proper preflight preparation including calibration and alignments of sensors during acceptance campaign and numerical simulations on test platforms to validate the mission and vehicle specific data for each flight.

\section{LESSONS LEARNED}

\subsection{Knowledge of Ionospheric Error for GPS Measurements}

During the far rendezvous, the ATV navigation (RGPS) and the FCM are based on GPS measurements difference provided by ATV and ISS GPS receivers. The knowledge of ionospheric error in terms of intensity and time evolution was a key 
element to survey along all ATV flights in order to confirm that predictions used for flight preparation and validation remained valid.

The different parameters which have an influence on ionospheric error level, are as follows:

- ISS altitude (the lower the altitude, the higher is the ionospheric error level): the altitude of ISS varied from $320 \mathrm{~km}$ for ATV-1 to $420 \mathrm{~km}$ for ATV-5;

- year of flight: solar activity has a cycle period of 11 years, 2008 (ATV-1 flight) was one of the lowest level, then solar activity increased to reach the maximum in 2012/2013 for ATV-3/4;

- month of flight: the worst case month for ionospheric error level is around April (ATV-1/2/3/4) then October;

- solar local time for rendezvous: worst case around $14 \mathrm{~h}$; and

- equator crossing event: higher ionospheric error level around the magnetic equator, lower level at high latitude.

After postflight analysis of ATV-1 flight, it appears that the numerical ionospheric error model used for qualification was sufficient for a flight in 2008 but not sufficiently representative to prepare and validate future ATV flights which have to be done in more severe conditions. The consequences were essentially identified in term of mission success probability reduction due to FCM false alarm rate increase and side effect on GPS satellites rejection by RGPS navigation filter.

For ATV production phase (ATV-2 to 5), the ionospheric error model was improved to be more representative by taking into account the higher level of ionospheric error which can be encountered for all ATV missions in the worst conditions for year, month, and local time but also by taking into account a variation of the level with respect to latitude. For the maximum level, the reference was issued from IRI90 model with $R Z=100$ for solar activity (Fig. 11). For the variation with latitude, the same principle of ICD (Interface Control Document) 200 for GPS concerning ionospheric error correction was used. Figure 12 shows the level pseudorange lengthening due ionospheric error with respect to elevation considered for ATV-1 and updated for ATV-2-ATV-5.

Validation on ATV numerical platforms using this new ionospheric error model allowed to justify that with a new tuning of RGPS filter rejection threshold, the ATV GNC performance was not affected by the ionosphere impact even in the worst case conditions. Furthermore, due to system margin for the FCM, the degradation of mission success associated to potential false alarm was negligible and the safety of ISS was not impacted. 


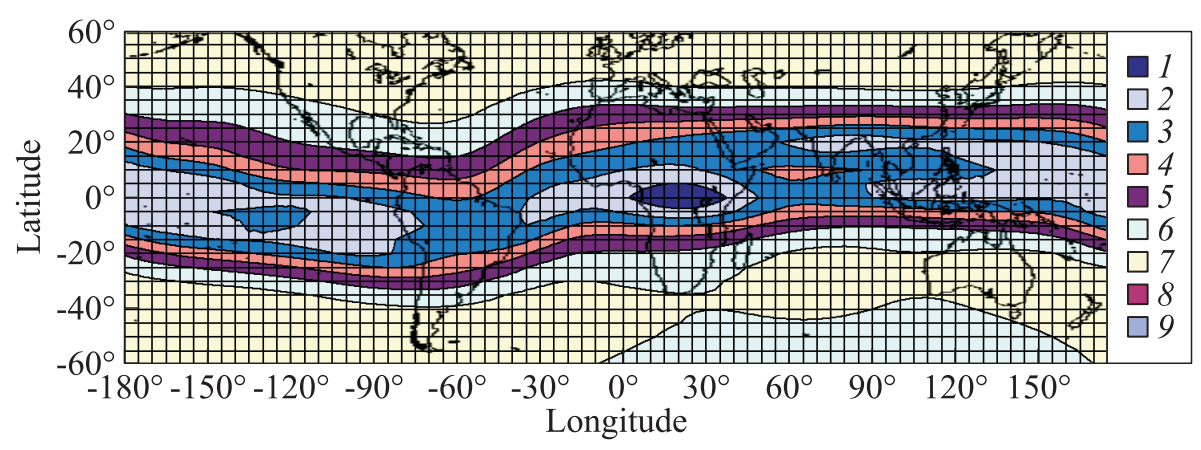

Figure 11 Pseudorange lengthening due to ionospheric error from IRI90, RZ=100, 14h30 local time: $1-16-18 \mathrm{~m} ; 2-14-16 ; 3-12-14 ; 4-10-12 ; 5-8-10 ; 6-$ $6-8 ; 7-4-6 ; 8-2-4$; and $9-0-2 \mathrm{~m}$

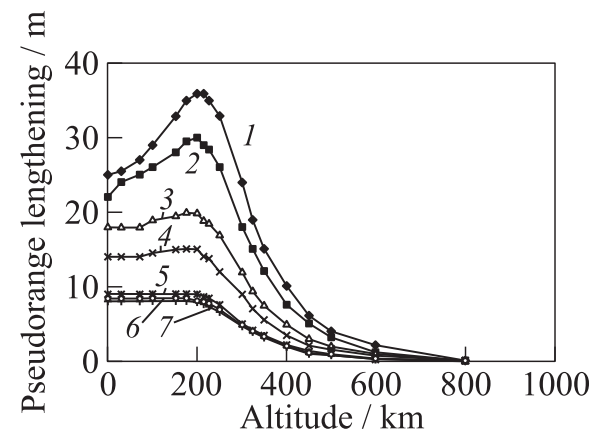

(a)

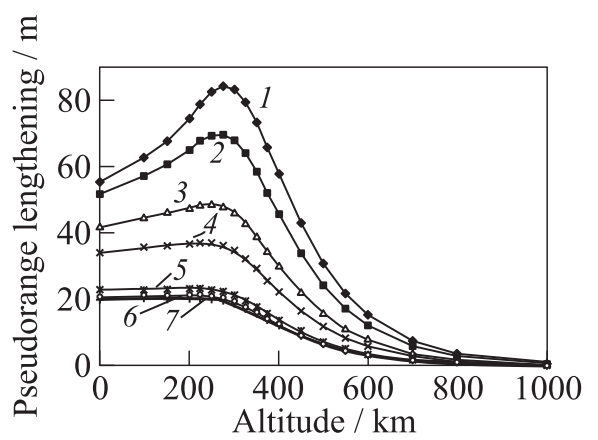

(b)

Figure 12 Pseudorange lengthening due to ionospheric error with resprect to altitude at different elevation used for ATV-1 $(a)$ and ATV-2-ATV-5 $(b): 1-5^{\circ} ; 2-10^{\circ}$; $3-20^{\circ} ; 4-30^{\circ} ; 5-60^{\circ} ; 6-75^{\circ}$; and $7-90^{\circ}$

All these assumptions on ionospheric error modeling were confirmed by the different ATV flights. Figure 13 shows the ionospheric error effect in term of difference between Pseudorange and Doppler Count measured by ATV receiver during the worst case obtained for ATV-3 (more than $50 \mathrm{~m}$ ).

Moreover, a high day to day variability of ionospheric error was observed during the different ATV missions which also justify the margins considered to build the ionospheric error numerical model used for ATV-2-ATV-5 with respect to IRI90 model prediction.

During all the ATV production, the ionosphere evolution and possible impact on the mission were constantly surveyed and challenged with respect to the hy- 




Figure 13 ATV-3 GPS Pseudorange minus Doppler Count between 30 and 10 degree of elevation: $1-10-20 \mathrm{~m} ; 2-20-30 ; 3-30-40 ; 4-40-50$; and $5->50 \mathrm{~m}$

pothesis taken for modeling. Therefore, the behaviors of RGPS and FCM using GPS difference were flawless ensuring performance, rendezvous success, and ISS safety without any false alarm caused by ionospheric error.

\subsection{Star Trackers Dazzled by Thrusters Plume}

Automated transfer vehicles used two star trackers for attitude navigation update and gyrometers drift estimation. The ATV attitude navigation is initialized by using information coming from Ariane 5 at separation after lift-off or coming from ISS before undocking. But in case of survival flight phase following onboard major failures detection, attitude navigation is initialized by using star tracker measurement in a "lost in space" mode. The ATV survival mode was never automatically triggered during any ATV flight, but this mode was tested for demonstration purpose on March 14, 2008 during the 1st ATV flight.

It was known that star trackers used by ATV (SED 16 SODERN) could be dazzled when a body such as Sun, Moon, or ISS enters in the field of view. But during all ATV flights, unexpected star tracker dazzling was also observed when some thruster plume illuminated by Sun or Moon entered in the field of view as shown in Fig. 14.

Depending on the dazzling level, the plumes of the thrusters could induce a loss of tracking. However, once initialized, ATV attitude navigation was robust to these temporarily losses without impact on performance. The only critical phase was the survival exit phase experienced on March 2008. The controller design was such that it commanded permanently a set of thrusters to perform an angular rate reduction followed by a specific sequence for star acquisition in order to initialize the attitude navigation. For ATV-1, this operation occurred during 


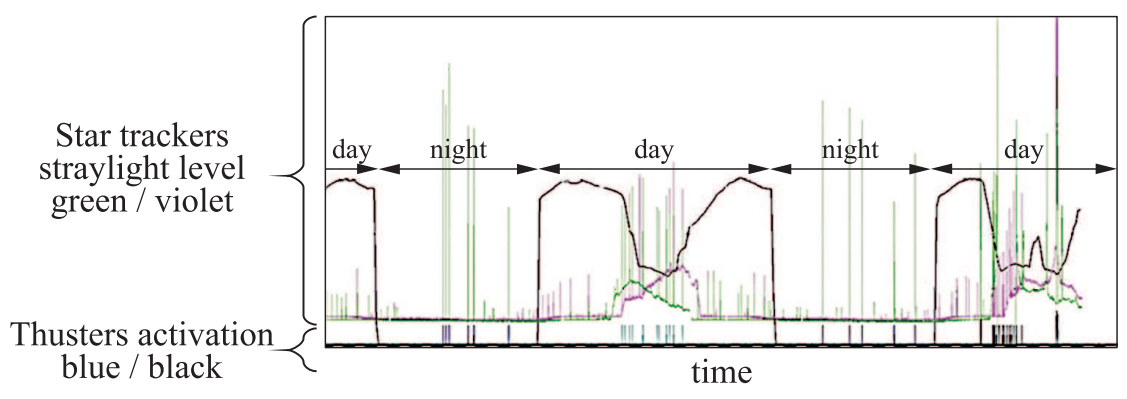

Figure 14 Star trackers dazzling over day/night and thruster activations

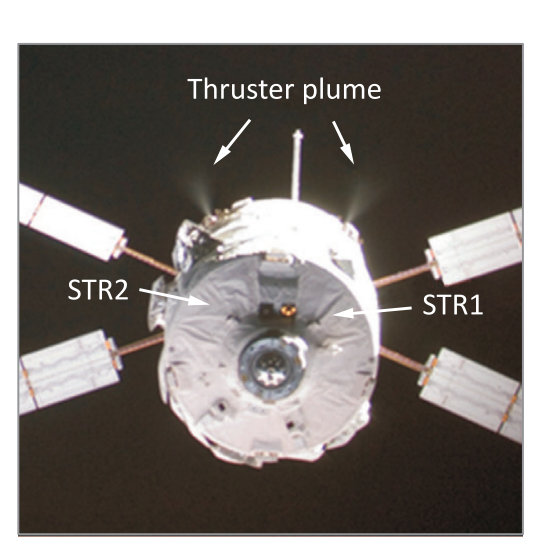

(a)

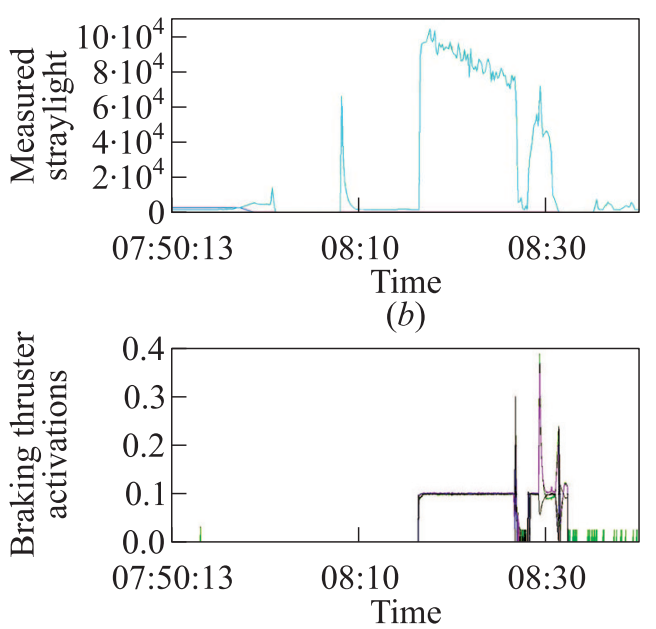

(c)

Figure 15 Thruster plume on ATV-1 approaching ISS (a), star trackers dazzling (b), and thruster activations $(c)$ on $14 / 08 / 2008$

daylight and, unfortunately, the selected thrusters dazzled both star trackers with the consequence to delay star trackers to go through tracking mode and, therefore, delaying the attitude navigation initialization (Fig. 15).

For ATV-1, there was no consequence for the demonstration, but considering other constraints like possible presence of bodies (Sun, Moon, or Earth) in the field of view or robustness to one star tracker failure, it was not possible to prove that in all cases, the exit of survival would be successful leading to the potential definitive loss of ATV. Therefore, the robustness of survival exit was improved 
by modifying GNC survival guidance and control. During the star acquisition, the thrusters activation was inhibited sequentially in order to avoid plume effect and then to allow the star trackers to go through the tracking mode.

To minimize the impacts, the possible side effects, and the requalification, the GNC algorithm was modified only during the star acquisition sequence when angular rate is stabilized. The duration $T_{\text {thrust }}$, when thrusters are activated, was chosen to allow the control of the vehicle, in accordance with the specifications. The duration $T_{\mathrm{str}}$, without thruster activation, was chosen in order to allow star tracker acquisition in the worst case situation without any parasite object in the field of view and maximum angular rate consistent with GNC capabilities. A specific validation demonstrated that in all configurations of Sun, Moon, Earth, and star tracker failure, the GNC performance in survival mode was conformed to specifications and attitude navigation was always correctly initialized. This GNC survival modification was implemented on ATV-3, but has never been used during remaining ATV flights.

\subsection{GPS Loss of Tracking due to Equatorial Anomaly Phenomena}

During ATV-3 postundocking free flight phase, a new observation was made on ATV GPS receivers tracking list. Sparse losses of tracking on several GPS satellites happened simultaneously, with a duration of maximum $90 \mathrm{~s}$ for situations involving less than 5 remaining satellites. Associated with the loss of tracking, cycle-slips, i.e., discontinuities in the GPS measurements, were also detected. Such phenomenon was not observed during ATV-2 flight, and looking back at ATV-1 telemetry data, only one occurrence of this phenomenon could be found.

The worst case observed during ATV-3 flight led to a complete tracking loss with no available satellite during at least $11 \mathrm{~s}$ on both GPS receivers. Because a test on available satellites number is performed by the Flight Application Software (FAS) to ensure healthiness of receivers, such event could have had an impact on ATV mission. A satellite is considered as unusable if a cycle slip appears or at least one raw data measurement over the two ones (Pseudorange or Doppler Count) is unavailable. During ATV rendezvous, the GPS receiver is declared failed after three occurrences $(3 \mathrm{~s})$ if no satellite is judged available by FAS. During free-flight, this test is disabled and, hence, there was no impact during this phase for ATV-3 mission. However, if such phenomenon would have appeared during rendezvous, potentially both GPS receivers could have been declared failed, which would automatically trigger an Escape manoeuvre and the abort of the rendezvous with ISS, while the navigation filters onboard ATV have been designed to handle unavailability of GPS measurements for durations larger than the ones of such events. 


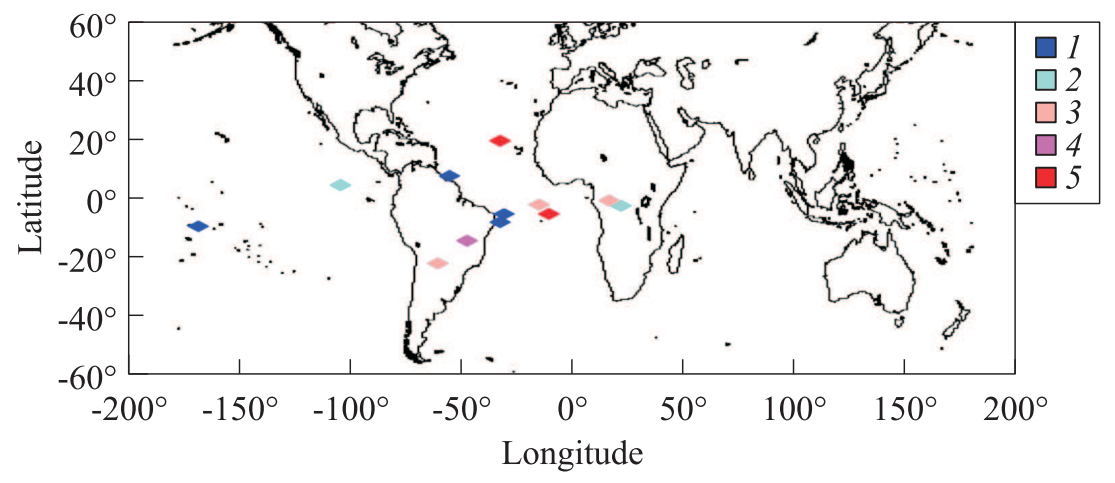

Figure 16 Loss of GPS satellites tracking during ATV-3 descent phase: $1->5$; $2-5 ; 3-4 ; 4-3$; and $5-<3$

Looking closer at the data collected during ATV-3 flight, it has been observed that these events always occurred for a local time, relative to the Sun position at a given longitude, between 19 and $01 \mathrm{~h}$. Also, when plotting the location of the losses of GPS reception on a map (Fig. 16), it can be seen that these events occurred when ATV crossed two given latitude bands around the equator. From the particular characteristics of these events, associated with high ionosphere, crossing magnetic equator in eclipse, local hour between 19 and 1, it was concluded that these could be attributed to the Appleton anomaly phenomena [9].

The Appleton or equatorial anomaly denotes the regions of enhanced plasma density from $10^{\circ}$ to $15^{\circ}$ in latitude north and south of the dip equator, occurring from prenoon through midnight hours. Appleton anomaly phenomena magnitude is linked to the level of ionosphere effect. In Fig. 17, there is an Earth map showing in red the geographical position of the enhanced plasma density during Appleton anomalies.

Further investigations on ATV-4 postundocking free-flight phase have been performed with an increased telemetry rate at $1 \mathrm{~Hz}$. The observed form of the perturbation is high-frequency noise on signal to noise ratio, sometimes very limited in time as seen in Fig. 18. Hence, it can be stated that the loss of tracking is associated to a scintillation phenomenon. The analysis showed that the perturbation affecting GPS reception is roughly higher than ATV, stays in the same place with respect to an Earth-Centered Earth-Fixed (ECEF) frame, is localized along a precise latitude line and affects the GPS satellites whose line of sight is precisely crossing that location line. Such observations confirmed the role of the Appleton anomaly in the observed GPS loss of tracking.

Since the potential impact of the environment on the capability of GPS receivers to track GPS satellites had not been considered, the simultaneous loss of 


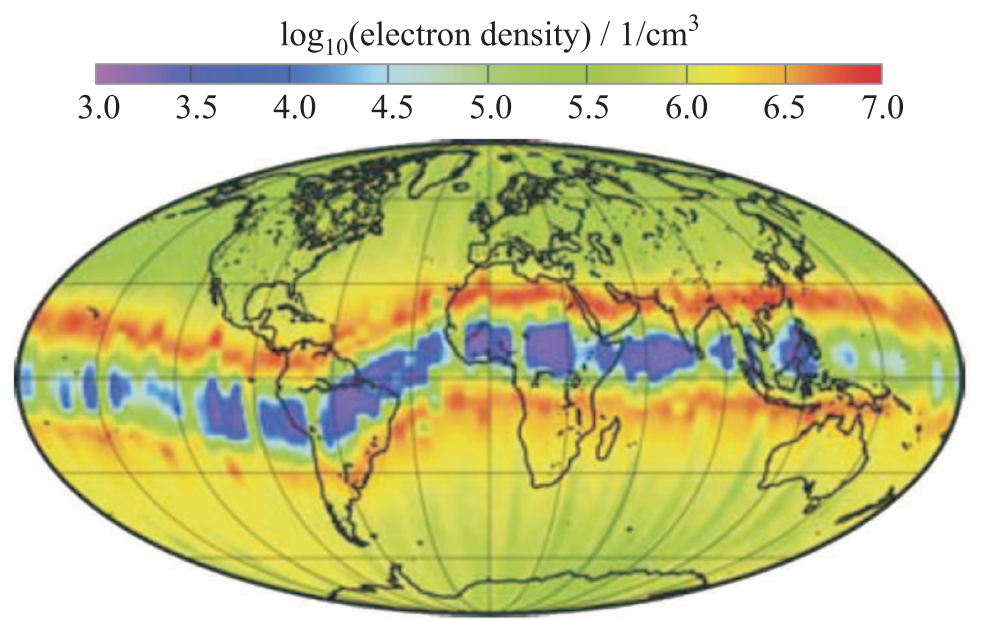

Figure 17 Longitude variation of electron density in 20 local time sectors [9]

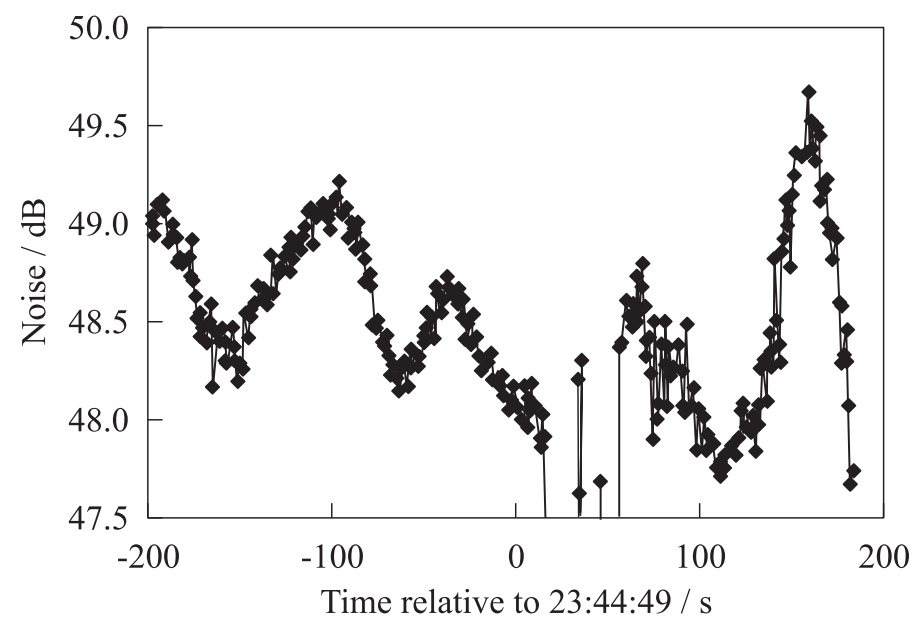

Figure 18 The ATV-4 GPS channel 1 signal to noise ratio at $1 \mathrm{~Hz}$ around an observed loss of tracking

tracking of all GPS satellites was associated to a failure of the GPS receivers. To avoid an interruption of the ATV mission associated to the Appleton anomaly, the local time during forecasted rendezvous was checked to be outside of the risk zone for ATV-4 and this failure detection mode suppressed on ATV-5 as already covered by other detection means not sensitive to this effect. 


\subsection{Inner Target Contamination of International Space Station}

During the last $20 \mathrm{~m}$ of the final approach to the ISS, ATV used the VDM to perform 6 degree of freedom relative navigation. The VDMs are imaging the 5 corner cubes of the inner target (Fig. 19) installed on ISS as seen in Fig. 3 to deduce the relative position and the relative attitude. The postflight analysis of the performance of ATV-3 navigation sensors revealed an abnormal behavior on relative attitude measurements. The yaw and pitch measurements for the redundant VDM (VDM-2) had been affected by errors well above the equipment specifications as illustrated in Fig. 20. Furthermore, VDM-2 lost tracking of the inner target several times. However, there was no abnormal behavior on all other measurements (position, roll, etc.) and all health status of the equipment was correct.

Further investigations have shown that the increased measurement noise was already encountered on ATV-2 but in a less severe way that did not lead to loss of tracking while for ATV-1, all measurements were within specifications.

Therefore, the analysis concluded that the inner target was likely affected by contamination especially on the central retroreflector that is used for pitch/yaw measurements. During previous ATV-2 undocking operations, the target may have been exposed to an oxidizer spill from the docking port refueling line that is used by ATV to deliver ISS propellant. The refueling line is close to the inner

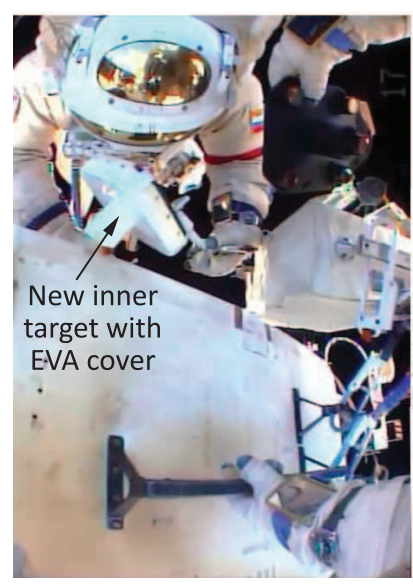

(a)

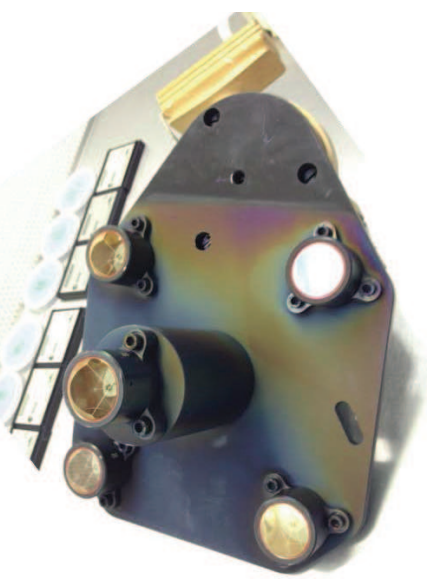

(b)

Figure 19 (a) Extra-vehicular activity to replace ATV inner target on ISS Service Module. New inner target is shown within its EVA protective cover while contaminated inner target retrived in ESA lab. is without cover $(b)$ 


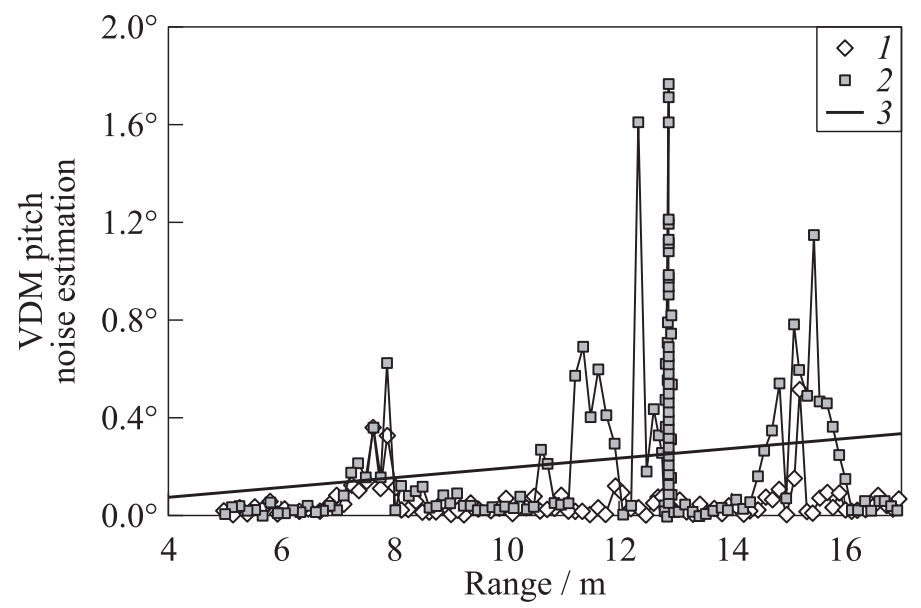

Figure 20 ATV-3 postflight analysis - VDMs pitch estimation noise: 1 - ATV-3 VDM-1; 2 - ATV-3 VDM-2; and 3 - space

target and some droplets of oxidizer were observed by crew being vented from ATV line toward ISS.

These facts prompted a decision for recovering and exchanging the inner target with a spare unit from the exterior of the ISS prior to attempt ATV4 rendezvous and docking. An Extra-Vehicular Activity (EVA) took place on April 19, 2013 (see Fig. 19) to perform the replacement operation. The old inner target that had already spent almost 9 years in space was recovered and stored within ISS and returned to Earth aboard Soyuz 34S on September 11, 2013.

The following docking operations of ATV-4 confirmed that the replacement of the inner target had been efficient in suppressing the measurements perturbations in yaw and pitch observed on ATV-2 and ATV-3. Meanwhile, ground investigations on the retrieved inner target [10] have shown that there was an orange contamination on the surface of the baseplate and on the optical surfaces with a varying thickness across the surface (see Fig. 19). No other damage from micrometeoroid or other was found.

Another interesting finding was made looking at Fig. $19 b$ where a clear transition between the contaminated area (orange) and the original black plasmoceramic coating of the baseplate appears above the retroreflectors. This demarcation line corresponds to the location of the EVA cover on top of the inner target which was used during ground operations, storage, and EVA. Similar images from the new inner target unit prior the flight have confirmed that a "preconditioning" deposit on the plasmoceramic coating as already been made preflight due to the EVA cover. No evidence of a proper baking of the EVA cover, to 


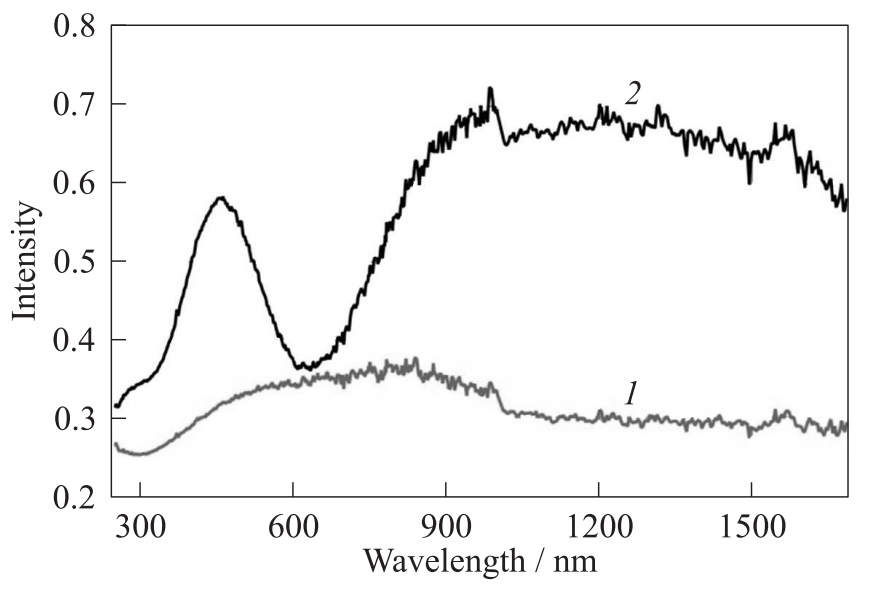

Figure 21 The VASE data comparing a reference ground retroreflector and a retroreflector recovered from the replaced ISS inner target: 1 - reference cube; and $2-$ ISS cube

remove volatiles and ensure absence of outgassing, prior to be in contact with the inner target was found during investigations.

Optical measurements on the inner target have also been performed to characterize the contamination. The overall total reflectivity of the retroreflectors was found not to be affected by the contamination at VDM wavelength. This result is consistent with flight observations that despite the contamination, the overall magnitude of the signal returned by the inner target from VDM/TGM is not significantly impacted.

Using Variable Angle Spectroscopic Ellipsometric (VASE) technique, the reflectivity of the front surface of the corner cubes of the inner target and properties of the layer deposited on these surfaces were estimated. The enhanced reflectivity of the replaced ISS inner target retroreflector is quite evident (Fig. 21). In particular, it can be seen that at the VDM operating wavelength $(811 \mathrm{~nm})$, the enhanced reflectivity is of about a factor 1.6. Furthermore, using the same technique, it is possible to estimate the optical constants of the contaminated layer and to deduce an estimated thickness. The estimated thickness of absorbing layer comes out to be $196 \mathrm{~nm}$ with a roughness of $3.6 \mathrm{~nm}$.

Therefore, two different surface effects have been detected during replaced ISS inner target inspection. One linked to a molecular contamination preflight (also present on the inner target currently on ISS) and the second one linked to ATVZvezda oxidizer refueling line that may have interacted. Further care should then be taken when dealing with optical target to ensure the proper material selection for low outgassing and proper baking of all elements in contact with the 
target should be ensured [10]. Finally, any optical target should preferably be located away or shielded from potential contamination sources such as refueling lines.

\section{CONCLUDING REMARKS}

This paper presents the main performances of the ATV GNC during rendezvous by comparing the dissimilar navigations performed onboard. Overall, all ATVs have delivered a performance much better than its specifications and with a great consistency among the various flights. This performance culminated in achieving for the last two ATVs a direct capture with ISS docking port without first touching the receiving cone. The 2-millimeter accuracy achieved by the fully automated docking ATV is an illustration of the European know-how developed in the frame of the ATV programme which has earned the ISS partners recognition.

Despite the achieved flawless dockings for the five missions, some observations and issues have arisen during the programme that required to be fixed in order to maintain mission success. The knowledge of the impact of the environment on RGPS navigation has been improved and refined following analysis performed in the frame of the postflight reviews. Observation of unforeseen dazzling of star trackers by thrusters plume led to modifications of survival mode to exclude potential interactions during this highly critical phase. And high perturbations on pitch and yaw measurements on VDM-2 during ATV-3 rendezvous led to an EVA to replace the ISS inner target that had been contaminated both preflight by its EVA cover and in-orbit by the spill of oxidiser from refueling lines during previous ATVs undocking.

\section{ACKNOWLEDGMENTS}

The authors of this paper would like to thank all the persons at the European Space Agency, the industrial prime Airbus Defence and Space and its subcontractors involved in the design, qualification, validation, operations, and postflight assessment of the ATV GNC. Their commitment and dedication all across the duration of the programme have been a key to the success of this programme.

\section{REFERENCES}

1. Personne, G., A. Lopez-y-diaz, and P. Delpy. 2005. ATV GNC synthesis: Overall design, operations and main performances. 6th ESA Conference (International) on Guidance, Navigation \& Control Systems. Greece. 
2. Cavrois, B., S. Chavy, P. Delpy, and S. Reynaud. 2005. ATV flight control monitoring: A dedicated ATV function to ensure the safety of the ISS during rendezvous. 1st IAASS Conference.

3. Mongrard, O., G. Bergez, B. Cavrois, and F. Martel. 2005. ATV on-board flight control monitoring design and trajectories safety assessment. 1st IAASS Conference.

4. Narmada. 2005. ATV far rendez-vous navigation based on relative GPS. 18th Technical Meeting (International) of The Satellite Division of The Institute of Navigation. Long Beach, CA. 1160-1166.

5. Cavrois, B., S. Reynaud, G. Personne, S. Chavy, and S. Strandmoe. 2008. ATV GNC and safety functions synthesis: Overall design, main performances and operations. AIAA Paper No. 2008-7481.

6. Mongrard, O., C. Santini, M. Powe, B. Cavrois, M. Zink, and A. Donnard. 2012. ATV GNC for rendezvous and docking: Principles, technology and experience. IAC-12.C1.2.1. $13 \mathrm{p}$.

7. Silva, N., F. Martel, and P. Delpy. 2005. Automated transfer vehicle thrusters selection and management function. 6th ESA Conference (International) on Guidance, Navigation and Control Systems. Greece.

8. Bourdon, J., P. Delpy, M. Ganet, I. Quinquis, and F. Ankersen. 2002. Application of $H_{\infty}$ design on ATV control loop during rendezvous phase. 5th ESA Conference (International) on Guidance, Navigation and Control Systems Proceedings. Frascati, Italy: ESA. 289.

9. Lühr, H., M. Rother, S. Maus, W. Mai, and D. Cooke. 2003. The diamagnetic effect of the equatorial Appleton anomaly: Its characteristics and impact on geomagnetic field modeling. Geophys. Rev. Lett. 30:1906.

10. Malicki, M., A. Bolkhovitinov, Y. Butenko, D. Doyle, O. Mongrard, and G. van Papendrecht. 2015. Characterisation of surface properties of laser retroreflector retrieved from the International Space Station. 13th Symposium (International) on Materials in the Space Environment. 